\title{
Purcell effect in Tamm plasmon structures with QD emitter
}

(C) A.R. Gubaydullin ${ }^{1,2}$, C. Symonds ${ }^{2}$, J. Bellessa ${ }^{2}$, K.A. Ivanov ${ }^{1,3}$, E.D. Kolykhalova ${ }^{1,4,5}$, M.E. Sasin ${ }^{4}$, G. Pozina ${ }^{6}$, M.A. Kaliteevski $i^{1,3,4}$

${ }^{1}$ St. Petersburg Academic University, Russian Academy of Sciences, 194021 St. Petersburg, Russia

2 Univ Lyon, Université Claude Bernard Lyon 1, CNRS, Institut Lumière Matiére, F-69622, LYON, France

${ }^{3}$ ITMO University,

197101 St. Petersburg, Russia

${ }^{4}$ loffe Institute,

194021 St. Petersburg, Russia

${ }^{5}$ St. Petersburg Scientific Center, 199034 St. Petersburg, Russia

${ }^{6}$ Department of Physics, Chemistry and Biology, Linköping University, 58183 Linköping, Sweden

E-mail: m.kaliteevski@mail.ru

We study Tamm plasmon structure based on $\mathrm{GaAs} / \mathrm{Al}_{0.95} \mathrm{GaAs}$ distributed Bragg reflector covered by thin silver layer, with active area formed by InAs quantum dots. We have measured the spectral and angular characteristics of photoluminescence and performed theoretical calculation of the spontaneous emission rate (modal Purcell factor) in the structure by using $S$-quantization formalism. We show that for Tamm plasmon mode the spontaneous emission can be enhanced by more than an order of magnitude, despite absorption in metallic layer.

\section{Acknowledgements}

The authors would like to thank A. Lemaitre and P. Senellart for the growth of the epitaxial structures. The work was supported by Russian Science Foundation grant N 16-12-10503. CS and JB acknowledge financial support from Agence Nationale de la Recherche (ANR) on ANR project NEHMESIS and FP7 project HyMeCav. GP acknowledge the Swedish Research Council (VR). KI acknowledge support Presidium of RAS grant 1.1.4.6 „Optoelectronic devices based on Tamm plasmons“. 